\title{
Comportamiento del cultivo del limón (Citrus aurantifolia Swingle) en "fincas tipo" en Santa Elena, Ecuador
}

\author{
Lemon crop (Citrus aurantifolia Swingle) behavior in farms \\ type of Santa Elena, Ecuador \\ Mercedes Santistevan Méndez ${ }^{1}$, Salomón Helfgott Lerner ${ }^{1}$, Oscar Loli Figueroa ${ }^{1}$, \\ Alberto Julca Otiniano ${ }^{*}$
}

\section{RESUMEN}

Se realizó una investigación entre agosto y noviembre del 2015, con el objetivo de conocer el comportamiento del cultivo de limón (Citrus aurantifolia Swingle) en "fincas tipo" de la provincia de Santa Elena, Ecuador. Una "finca tipo" es representativa de un grupo de fincas con características socioeconómicas similares. Se seleccionaron cuatro "fincas tipos" con cultivo de limón de ocho años de edad. En cada finca se marcaron 50 plantas, en las que se hicieron las evaluaciones. El ensayo no tuvo un diseño experimental, pero el análisis estadístico se hizo como si fuera un Diseño Completamente al Azar (DBCA), considerando cada finca como un tratamiento y cada planta como una repetición. Se encontró que la mayor incidencia de pulgones (Aphis spp.) correspondió a la finca tipo I, estadísticamente diferente a la finca IV pero similar a las fincas II y III. La mayor incidencia de mosca blanca (Aleurothrixus floccosus), correspondió a la finca tipo I, estadísticamente diferente a las fincas III y IV. La mayor incidencia de fumagina (Capnodium sp.), correspondió a la finca tipo I, pero estadísticamente similar a las otras fincas. El mayor diámetro y peso del fruto, correspondió a la finca III, estadísticamente mayor a las fincas I y II pero similar a la finca IV.

Palabras clave: tipología, fincas, cítricos, plagas, calidad.

\begin{abstract}
An investigation was carried out between August and November 2015 in order to understand the behavior of the lemon crop (Citrus aurantifolia Swingle) in "farm types" in the Province of Santa Elena, Ecuador. A "farm type" represents a group of farms with similar socio-economic characteristics. Four eight years old "farms types" were selected. In each farm, 50 plants were marked in which assessments were made. The trial did not have an experimental design but statistical analysis was done like a Completely Randomized Design (CRD), considering each farm type as a treatment and each plant as a replicate. The highest aphid (Aphis spp.) incidence was found in type I farms. Values were statistically different from type IV farms but similar to farm types II and III. The highest incidence of whitefly (Aleurothrixus floccosus), was found in type I farms that were statistically different from farm types III and IV. The highest incidence of sooty mold (Capnodium sp.), was found in type I farm that were statistically similar to other farms. The largest diameter and fruit weight, was found in farm type III with values that were statistically higher than farm types I and II but similar to farm type IV.
\end{abstract}

Key words: typology, farms, citrus, pests, quality.

\section{Introducción}

El cultivo de cítricos a nivel mundial es muy importante. En Ecuador, el limón Sutil (Citrus aurantifolia Swingle) es la especie más cultivada, seguida del limón Tahití (Citrus latifolia Tan), entre ambos suman aproximadamente 4.400 ha cultivadas. En la provincia de Santa Elena, hay 500 ha de limón sutil, asociadas mayormente con pequeños productores. Un estudio elaborado por Proyecto Integral de Desarrollo Agrícola Ambiental y Social de forma sostenible en Ecuador, determinó que en

\footnotetext{
1 Universidad Nacional Agraria La Molina (UNALM). Apto. 12056. La Molina. Lima, Perú.

* Autor para correspondencia: ajo@lamolina.edu.pe
}

Fecha de Recepción: 29 Octubre, 2016.

Fecha de Aceptación: 16 Enero, 2017.

DOI: $10.4067 / \mathrm{S} 0718-34292017005000003$ 
la provincia de Santa Elena el área cultivada con limón, está distribuida en unidades de producción agropecuaria que tienen un área entre 0,3 a 10 ha; a esta actividad se dedican unos 400 agricultores (PIDASSE, 2011). Frecuentemente, en reuniones con técnicos y agricultores de la provincia, se ha sugerido la necesidad de realizar investigaciones, para ayudar a la mejora tecnológica de este cultivo en la zona porque el rendimiento es muy variable entre una localidad y otra y entre las fincas de la misma localidad. Hart (1990), citado por Malagon y Prager (2001), define una finca como uno de los niveles de la jerarquía de sistemas agrícolas. Santistevan et al. (2015), en la provincia de Santa Elena, encontraron que existen fincas productoras de limón que producen menos de 10 toneladas (16\%), 10-15 t/ha (20\%), 16-25 t/ha (47\%), 26-30 t/ha (9\%) y más de 30 t/ha (8\%). En las Tablas 1 y 2 se describen las fincas de esta provincia, clasificadas en cuatro grupos (Santistevan et al., 2015). El primer grupo aglutina al 44,6\% de fincas, un segundo grupo al $15,7 \%$, el tercero al $26,5 \%$ y el cuarto grupo al $13,3 \%$. No se tiene información documentada sobre los rendimientos que tiene cada grupo, una forma de saberlo es estudiando una finca seleccionada en cada grupo y que se denomina "fincas tipo", estas son representativas del grupo del que fue seleccionado.
Estudios usando "fincas tipo", han sido realizadas por Salazar (2012), Tuesta et al. (2014) y Collantes (2016). Este trabajo de investigación se realizó con el objetivo de conocer el comportamiento del cultivo de limón en "fincas tipo" de la provincia de Santa Elena, Ecuador.

\section{Materiales y Métodos}

El estudio se realizó entre agosto y noviembre del 2015, para ellos se seleccionaron cuatro "fincas tipo" productoras de limón de ocho años de edad, en la provincia de Santa Elena, en cada una de estas, se marcaron 50 plantas, en las que se realizaron las evaluaciones respectivas. Previamente se tomó muestras del suelo de cada zona, las mismas que fueron enviadas al Laboratorio de Suelos de la Estación Experimental INIAP Boliche en el Guayas, Ecuador, las variables evaluadas fueron:

- Incidencia de plagas y enfermedades. La incidencia de las principales plagas y enfermedades se evaluó mensualmente durante los cinco meses que duró la investigación.

- Rendimiento. Se hicieron un total de 16 cosechas ("pasadas"), es decir, una vez por semana y los datos se procesaron para cada mes.

Tabla 1. Características más importantes de "fincas tipo" productoras de limón en Santa Elena, Ecuador.

\begin{tabular}{|c|c|c|c|c|}
\hline Características & Tipo I & Tipo II & Tipo III & Tipo IV \\
\hline Cantidad que representa (\%) & 44,6 & 15,7 & 26,5 & 13,3 \\
\hline Promedio área total (ha) & 2,3 & 4,3 & 4,5 & 16,5 \\
\hline Promedio área con limón (ha) & 1,2 & 1,2 & 2,5 & 4,5 \\
\hline Promedio $\mathrm{N}^{\circ}$ plantas/ha & 250 & 275 & 260 & 280 \\
\hline Inversión/ha/año (US\$) & 325 & 400 & 625 & 1.450 \\
\hline Ingreso mensual/ha (US\$) & 450 & 540 & 675 & 700 \\
\hline Localidad & Manglaralto & Colonche & Colonche & Manglaralto \\
\hline \multirow[t]{2}{*}{ Ubicación geográfica } & $80^{\circ} 41^{\prime} 31,1994$ ' W & $80^{\circ} 41^{\prime} 29,5188^{\prime \prime} \mathrm{W}$ & $80^{\circ} 39^{\prime} 50,673^{\prime \prime} \mathrm{W}$ & $80^{\circ} 41^{\prime} 29,5188^{\prime \prime} \mathrm{W}$ \\
\hline & $1^{\circ} 56$ '14,2794" S & $2^{\circ} 0{ }^{\prime} 38,6064 " \mathrm{~S}$ & $1^{\circ} 53$ '44,00226" S & $1^{\circ} 56^{\prime} 8,685^{\prime \prime} \mathrm{S}$ \\
\hline
\end{tabular}

Fuente: Santistevan et al., 2015.

Tabla 2. Características del suelo en "fincas tipo" en la provincia de Santa Elena, Ecuador.

\begin{tabular}{lcccccc}
\hline Tipo de finca & $\mathrm{pH}$ & $\begin{array}{c}\mathrm{N} \\
(\mathrm{ppm})\end{array}$ & $\begin{array}{c}\mathrm{P} \\
(\mathrm{ppm})\end{array}$ & $\begin{array}{c}\mathrm{K} \\
(\mathrm{meq} / 100 \mathrm{ml})\end{array}$ & $\begin{array}{c}\mathrm{Ca} \\
(\mathrm{meq} / 100 \mathrm{ml})\end{array}$ & $\begin{array}{c}\mathrm{Mg} \\
(\mathrm{meq} / 100 \mathrm{ml})\end{array}$ \\
\hline Finca I & 7,5 & 24 & 33 & 2,32 & 18,02 & 4,75 \\
Finca II & 7,2 & 16 & 27 & 2,48 & 18,98 & 4,53 \\
Finca III & 7,1 & 28 & 15 & 0,83 & 17,52 & 6,41 \\
Finca IV & 7,1 & 20 & 44 & 2,26 & 18,42 & 7,28 \\
\hline
\end{tabular}

Fuente: Santistevan et al., 2015. 
- Calidad del limón. En cada una de las 16 cosechas ("pasadas"), del total de frutos, se tomó una muestra al azar de 10 frutos a los que se tomó el peso y el diámetro usando una balanza analítica y un vernier, respectivamente. Los datos se procesaron para cada mes.

El ensayo no tuvo un diseño experimental; pero el análisis estadístico se hizo como si fuera un Diseño Completamente al Azar (DBCA), considerando cada "finca tipo" como un tratamiento y cada planta de limón como una repetición. Una "finca tipo" es representativa de un grupo con características socioeconómicas similares, en la zona de estudio se determinaron la presencia de cuatro grupos diferentes de fincas (Santistevan et al., 2015) y las características principales se presentan en la Tabla 1.

\section{Resultados y Discusión}

Según Hart (1990), citado por Malagon y Prager (2001), las fincas son sistemas con diferentes recursos, procesos y componentes de producción, que los agricultores individuales o colectivamente, combinan para formar subsistemas que convierten recursos en productos. En este estudio, las "fincas tipo" se caracterizan por tener suelos mayormente de textura franca, con $\mathrm{pH}$ cercano a neutro, $\mathrm{N}$ entre bajo y medio; pero con alto nivel de $\mathrm{P}, \mathrm{K}, \mathrm{Ca}$ y $\mathrm{Mg}$, este último es mayor en los últimos tipos (Tabla 2).

\section{Incidencia de plagas y enfermedades}

Se reportó la presencia de pulgones (Aphis spp.), mosca blanca (Aleurothrixus floccosus) y fumagina (Capnodium sp.) problemas sanitarios muy comunes en limón y otras especies cultivadas de cítricos, tanto en Ecuador (Falconí, 1999; INIAP, 2002) como en otros países productores como el Perú (Vegas y Narrea, 2011). La mayor incidencia de pulgones, se presentó en la finca tipo I y la más baja correspondió a la finca tipo III, diferencia que fue estadísticamente significativa. La mosca blanca, tuvo su mayor incidencia en la finca tipo I y la más baja correspondió a la finca tipo IV; pero esta fue estadísticamente similar a la finca tipo III. La fumagina tuvo su mayor incidencia en la finca tipo I y la más baja correspondió a la finca tipo IV, pero no hubo diferencias estadísticamente significativas entre fincas (Tabla 3). En general, las
Tabla 3. Incidencia de plagas y enfermedades (\%) en "fincas tipo" en la provincia de Santa Elena, Ecuador.

\begin{tabular}{llcr}
\hline Tipo de finca & Pulgones & Mosca blanca & Fumagina \\
\hline Finca I & $26,0 \mathrm{a}$ & $25,0 \mathrm{a}$ & $35,0 \mathrm{a}$ \\
Finca II & $21,4 \mathrm{ab}$ & $18,7 \mathrm{ab}$ & $29,3 \mathrm{a}$ \\
Finca III & $16,0 \mathrm{~b}$ & $16,0 \mathrm{~b}$ & $24,0 \mathrm{a}$ \\
Finca IV & $18,6 \mathrm{~b}$ & $12,6 \mathrm{~b}$ & $20,0 \mathrm{a}$ \\
\hline
\end{tabular}

Nota: Prueba de Duncan al 95\% para prueba de medias entre fincas.

fincas tipo I y II, presentaron frecuentemente la presencia de malezas y estas pueden ser hospederos de plagas y enfermedades (Gobierno del Estado de Chiapas, 2013).

La presencia de estas plagas y enfermedades, corrobora la importancia económica de estas y la necesidad de diseñar adecuadas medidas de control, caso contrario podrían afectar negativamente a la cantidad y calidad de la cosecha. El deficiente control de plagas y enfermedades son determinantes en la baja producción y calidad de la fruta, lo que genera una baja rentabilidad del cultivo de limón (Vegas y Narrea, 2011).

\section{Calidad del fruto del limón}

Se consideran varios aspectos para la calidad del fruto; sin embargo, en la investigación solo se tomó en cuenta diámetro y peso del fruto.

Los productores de limón asocian la calidad del fruto generalmente con el tamaño; sin embargo, para la calidad de fruto se toma en cuenta varias variables: peso, diámetro ecuatorial y polar del fruto, grosor de la cáscara, el peso y el porcentaje de jugo, la acidez, los sólidos solubles y el contenido de vitamina C. Dorado, et al. (2015). En la investigación El diámetro varió en el tiempo y de una finca a otra, en el mes de agosto el fruto tuvo un mayor diámetro en la finca III y la más baja en la finca I, diferencias que fueron estadísticamente significativas. En la segunda evaluación, el fruto tuvo un diámetro significativamente mayor en la finca III y la más baja en la finca I que, a su vez, fue estadísticamente similar al resto de tratamientos. En octubre, el diámetro fue mayor en la finca III y la más baja en la finca I que, a su vez, fue estadísticamente similar al resto de tratamientos. En la última evaluación, el fruto tuvo un diámetro significativamente mayor en la finca III y la más baja en la finca I, diferencias que fueron estadísticamente significativas (Tabla 4). 
Tabla 4. Diámetro del fruto $(\mathrm{cm})$ en fincas tipo en la provincia de Santa Elena, Ecuador.

\begin{tabular}{lccccc}
\hline Tipo de finca & Agosto & Septiembre & Octubre & Noviembre & Promedio \\
\hline Finca I & $2,59 \mathrm{c}$ & $3,12 \mathrm{~b}$ & $3,29 \mathrm{~b}$ & $3,39 \mathrm{~b}$ & $3,09 \mathrm{c}$ \\
Finca II & $3,09 \mathrm{~b}$ & $3,24 \mathrm{~b}$ & $3,38 \mathrm{~b}$ & $3,46 \mathrm{~b}$ & $3,29 \mathrm{bc}$ \\
Finca III & $3,56 \mathrm{a}$ & $3,76 \mathrm{a}$ & $3,62 \mathrm{a}$ & $3,71 \mathrm{a}$ & $3,66 \mathrm{a}$ \\
Finca IV & $3,46 \mathrm{a}$ & $3,40 \mathrm{~b}$ & $3,45 \mathrm{ab}$ & $3,55 \mathrm{ab}$ & $3,46 \mathrm{a}$ \\
\hline
\end{tabular}

Nota: Prueba de Duncan al 95\%, para prueba de medias entre fincas.

El rango de valores encontrados en este estudio $(2,59-3,76 \mathrm{~cm})$ son cercanos a los reportados en otras investigaciones en el Ecuador, como el de Orrala et al. (2010), que encontró valores entre 3,63 y $3,86 \mathrm{~cm}$ en estudio realizado en Santa Elena. También a los reportados en México, que para limón criollo se encontró diámetros entre 3,66 ya $3,72 \mathrm{~cm}$ (Pérez, 2002).

Cuando se evaluó el peso del fruto, se encontró que también varió con el tiempo y de una finca a otra. En el mes de agosto el fruto tuvo un mayor peso en la finca IV y la más baja en la finca I, todos los tratamientos tuvieron diferencias estadísticas significativas entre sí. En la segunda evaluación, el fruto tuvo un peso significativamente mayor en la finca IV y la más baja en la finca I, la misma que fue estadísticamente diferente al resto de tratamientos en estudio. En octubre, el peso fue mayor en la finca IV y la más baja en la finca I, que, a su vez, fue estadísticamente similar a la finca II. En la última evaluación, el fruto tuvo un peso significativamente mayor en la finca III y la más baja en la finca I, diferencias que fueron estadísticamente significativas (Tabla 5). El rango de valores encontrados en este estudio (34,15 a 58,86 ) es superior al reportado en otros estudios realizados en el Ecuador, como el de Orrala et al. (2010), que encontró valores entre 28,29 y 35,01 g en Santa Elena. Superior a los encontrados en México, donde se reportó pesos de 32,2 a 34,8 g (Pérez, 2002) y entre 32,2 a 38,2 (Álvarez et al., 2008).

\section{Rendimiento del cultivo}

El mayor peso/parcela se reportó en la finca III $(2.730 \mathrm{~kg})$, la diferencia con las otras fincas evaluadas fue notoria, ya que el rango estuvo entre 752,5 a $2.730 \mathrm{~kg}$. Lógicamente, está tendencia fue la misma cuando se evaluó el rendimiento por unidad de área, donde la finca III con 13,7 t/ha tiene un rendimiento 3,6 veces superior, con respecto a la finca I que tuvo el peso más bajo (Tabla 6). Los valores alcanzados en estas fincas son bajos si los comparamos con otros reportes, como en México, donde el rendimiento promedio es de 12,5 t/ha (Caamal, et al. 2014); mientras que en Piura (Perú) está entre 9 y 14 t/ha (Vegas y Narrea, 2011). Los rendimientos por planta $(15,05-54,60 \mathrm{~kg})$, también son bajos, si lo comparamos con los $73,8 \mathrm{~kg} / \mathrm{pl}$ reportado por Orrala et al. (2010) en Ecuador.

Tabla 6. Rendimiento de limón en fincas tipo en la provincia de Santa Elena, Ecuador.

\begin{tabular}{lrrr}
\hline Tipo de finca & $\mathrm{Kg} /$ planta & $\mathrm{Kg} /$ parcela $^{1}$ & ${\mathrm{t} / \mathrm{ha}^{2}}$ \\
\hline Finca I & 15,05 & $752,5 \mathrm{~b}$ & 3,80 \\
Finca II & 22,05 & $1102,5 \mathrm{~b}$ & 6,06 \\
Finca III & 54,60 & $2730,0 \mathrm{a}$ & 14,19 \\
Finca IV & 45,85 & $2292,5 \mathrm{a}$ & 12,84 \\
\hline
\end{tabular}

${ }^{1}$ Prueba de Duncan al 95\%, para prueba de medias entre fincas. ${ }^{2}$ Estimados con 250, 275, 260 y $280 \mathrm{pl} / \mathrm{ha}$, para la finca I, II, III y IV, respectivamente.

Tabla 5. Peso del fruto (g) en fincas tipo en la provincia de Santa Elena, Ecuador.

\begin{tabular}{lccccc}
\hline Tipo de finca & Agosto & Septiembre & Octubre & Noviembre & Promedio \\
\hline Finca I & $35,05 \mathrm{~d}$ & $34,15 \mathrm{c}$ & $35,77 \mathrm{c}$ & $35,03 \mathrm{c}$ & $35,0 \mathrm{c}$ \\
Finca II & $40,85 \mathrm{c}$ & $42,42 \mathrm{~b}$ & $40,79 \mathrm{c}$ & $42,01 \mathrm{bc}$ & $41,52 \mathrm{~b}$ \\
Finca III & $48,71 \mathrm{~b}$ & $47,18 \mathrm{ab}$ & $48,95 \mathrm{~b}$ & $58,86 \mathrm{a}$ & $50,92 \mathrm{a}$ \\
Finca IV & $53,54 \mathrm{a}$ & $49,20 \mathrm{a}$ & $54,77 \mathrm{a}$ & $47,89 \mathrm{~b}$ & $51,35 \mathrm{a}$ \\
\hline
\end{tabular}

Nota: Prueba de Duncan al 95\%, para prueba de medias entre fincas. 
Si se considera que las dos zonas productoras de limón más importantes en la provincia de Santa Elena (Manglaralto y Colonche), tienen condiciones climáticas bastante parecidas (INHAMI, 2014), la diferencia de resultados entre una finca y otra, aunque no siempre estadísticamente significativa, se explicaría por un entorno económico diferente (Tabla 1) y las características personales del propietario de cada finca. Según Hart (1990), citado por Malagon y Prager (2001), los sistemas de fincas son producto de tres fuerzas generales: el ambiente físico-biológico, el ambiente socioeconómico y las habilidades del productor. Los resultados, muestran que existe una brecha que es necesario acortar, especialmente si tenemos en cuenta que la finca tipo I representan al 44,6\% de las unidades productoras de esta zona de estudio. En el futuro sería recomendable trabajar en la mejora de la producción de este cultivo, tarea en la que el productor de limón debe tener un rol protagónico.

\section{Conclusiones}

En general se observa ue los pulgones y la fumagina, afectan a todos los tipos de fincas. Sin embargo, en el caso del pulgón, respecto a la mosca blanca, se observa una menor incidencia en las fincas con mayor inversión por año. La producción total mejora en las fincas tipo III y IV que son estadísticamente mayores que las fincas tipo I y II debido, principalmente, al mayor diámetro y peso de frutos.

\section{Literatura Citada}

Álvarez, A.; Saucedo, C.; Sergio Chávez, S.; Víctor Medina, V.; Colinas, M.; Báez, R.

2008. Reguladores de crecimiento en la maduración y senescencia de frutos de limón mexicano. Agricultura Técnica en México, 34 (1): 5-11.

Caamal, I.; Pat, V; Ascencio, F.; Santoyo, L., y Ramos, J. 2014. Análisis de los costos de producción del limón persa en el municipio de Tlapacoyan, Veracruz. Revista Científica Biológico Agropecuaria Tuxpan, 2 (3): 192-200.

Dorado, D.; Grajales, L. y Ríos L.

2015. Efecto del riego y la fertilización sobre el rendimiento y la calidad de la fruta de lima ácida Tahití Citrus latifolia Tanaka (Rutaceae). Corpoica Ciencia Tecnología Agropecuaria, 16 (1): 87-93.

Falconí, C.

1999. Fitopatógenos. Enfermedades, plagas, malezas y nemátodos fitopatógenos de cultivos en el Ecuador. Centro de Diagnóstico y Control Biológico. Universidad San Francisco de Quito, Quito, Ecuador, 123 p.

Gobierno del Estado de Chiapas

2013. Secretaria de Desarrollo Social Proyecto Desarrollo Social Integrado y Sostenible. Malezas en el cultivo del limón, Chiapas, México, 36 p.

Instituto Nacional Autónomo de Investigaciones Agropecuarias - INIAP.

2002. Informe técnico anual. Sección Entomología. Estación Experimental Portoviejo, $21 \mathrm{p}$.

Instituto Nacional de Meteorología e Hidrología - INAMHI. 2014. Cambio Climático. Detección de cambio climático en el Ecuador. Disponible en: http://www.serviciometeorologico. gob.ec/cambio-climatico/ Consultado: 5/ mayo/2016.

Malagon, R; Praguer, M.

2001. El enfoque de sistemas: Una opción para el análisis de las unidades de producción agrícola. Universidad Nacional de Colombia. Palmira, Colombia, 190 p.
Ministerio de Agricultura, Ganadera, Acuacultura y Pesca.

2011. Proyecto Integral de Desarrollo Agrícola Ambiental y Social de Forma Sostenible en el Ecuador - PIDAASSE. 2011. Resultados Proyecto PIDAASSE, 2010-2011. Quito, Ecuador.

Orrala, N.; Solís, L. y Tomalá, M.

2010. Efecto de NPK en la producción de Citrus Aurantifolia Swingle V. Sutil en la zona de Sinchal - Barcelona, Cantón Santa Elena. Revista Científica y Tecnológica. UPSE, 1 (1): $1-5$.

Pérez, $\mathrm{O}$.

2002. Evaluación de mejoradores del suelo en limón mexicano. Terra Latinoamericana, 20 (3): 337-346.

Santistevan, M.; Julca, A. y Helfgott, S.

2015. Caracterización de las fincas productoras del cultivo limón en las localidades de Manglaralto y Colonche (Santa Elena, Ecuador). Revista Científica y Tecnológica UPSE, 3 (1): 133-142.

Tuesta, O.; Julca, A.; Borjas, R.; Rodríguez, P. y Santistevan, M. 2014. Ttipología de fincas cacaoteras en la subcuenca media del río Huayabamba, distrito de Huicungo (San Martín, Perú). Ecología Aplicada, 13 (2): 71-78.

Vegas, U. y Narrea, M.

2011. Manejo integrado del cultivo de limón. Jornada de Capacitación. Universidad Nacional Agraria La Molina - AGROBANCO. Lima, Perú, 43 p.

Collantes, R.

2016. Sustentabilidad de los agroecosistemas de palto (Persea americana MiLL.) y mandarina (Citrus spp.) en Cañete, Lima, Perú. Revista Tecnología y Desarrollo, 13 (1): 027-034.

Salazar, R.

2012. Caracterización de sistemas agroecológicos para el establecimiento comercial de cacao orgánico (Theobroma cacao) en Talamanca. Revista Tecnología en Marcha, 25 (5): 45-54. 
